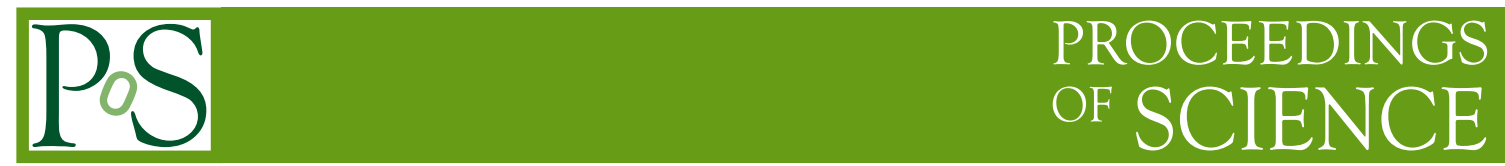

\title{
Radio supernovae
}

\author{
R. J. Beswick* \\ Jodrell Bank Observatory, The University of Manchester, Lower Withington, Nr. Macclesfield, \\ Cheshire, SK11 9DL, UK \\ E-mail: Robert.Beswick@manchester.ac.uk
}

I will briefly review the current status of radio observations of nearby supernovae and their remnants. This review will initially address observations of the radio 'light' curves of nearby corecollapse supernovae, followed by a more detailed summary of recent Very Long Baseline Interferometric observations of the expansion of nearby supernovae and their remnants. These later sections will concentrate on a few sources, namely those found in M82, SN1993J, and the recent supernova 2004et. In addition, I will discuss the many radio detections of supernovae found in the highly obscured centres of starburst galaxies, such as M82 and Arp220, where no optical detections are possible.

The 8th European VLBI Network Symposium

September 26-29, 2006

Toruń, Poland

${ }^{*}$ Speaker. 


\section{Introduction}

Radio supernovae and their remnants not only provide a tracer of the ongoing star-formation within galaxies but also, via radio 'light' curve monitoring and VLBI imaging, allow the physics of the surrounding circumstellar and interstellar media to be investigated. As such, the study of supernovae at radio wavelengths is complimentary to observations made at other wavelengths, whilst in all but the closest supernovae events, such as SN1987A, radio observations and in particular VLBI observations, provide the only method by which the structural evolution of supernovae can be directly imaged.

In many nearby galaxies with high star-formation rates, such as M82 and Arp220, numerous


incidentally is were a large amount of the Universe's star-formation is occurring, the extinction free nature of radio observations, mean that they are the only method by which these regions can be studied. With the high angular resolution and high sensitivity of radio interferometric observations, it is possible to detect, resolve and characterise the nature of these compact sources. For example, in M82, approaching 50 compact objects are detected at centimetric wavelengths; the majority of these sources are identified as supernova remnants, the remaining $\sim \frac{1}{3}$ are compact HII regions [6]. Sources like M82 and Arp220 provide an illustrative example of why radio observations are a vital addition to the numerous studies of supernovae at other wavelengths. In these two cases, as well as several others (e.g. IIIZw35 [7], Arp299 [8], Mrk 273 [9, 10], NGC6240 [11] etc.), radio observations, are the only way in which individual supernovae and their consequent remnants can be directly identified. Without such radio observations many supernovae within the highly obscured centres of galaxies may have been overlooked.

\section{Optical classifications of supernovae and optically detected number counts}

\subsection{Supernovae taxonomy}

Whilst this short review will exclusively concentrate upon radio observations and in particular high-resolution observations, it is useful to briefly recap the optical classifications and definitions of supernovae. Supernovae are optically classified into Type-II or Type-I objects depending on the detection or non-detection of hydrogen emission lines in early spectra, respectively. These fundamental categories are often further sub-divided. In the case of Type-I supernovae, these are traditionally split into Ia, Ib and Ic on the basis of their optical emission lines. Type-II supernovae are similarly classified into various groups on the basis of their optical spectra and light curve shape. These classes and their subsequent sub-divisions are schematically shown for the most common cases in Fig. 1. These classifications can be related to the physical mechanisms undergone within the supernova and in the star's pre-supernova phase.

In general, Type-Ia supernovae are thought to originate from the thermonuclear burn down of material accreted onto the surface of a white dwarf companion of a more massive giant star (see [12, 13] and references therein). As such, these Type-Ia supernova are not core-collapse type in origin and are analogous to galactic nova sources, for example RS Ophiuchi, which is thought may eventually become a Type-Ia supernova (see [14, 15, 16]). Type-Ia supernovae are often considered as relatively homogeneous events in the optical with similar luminosities and spectral evolution, 


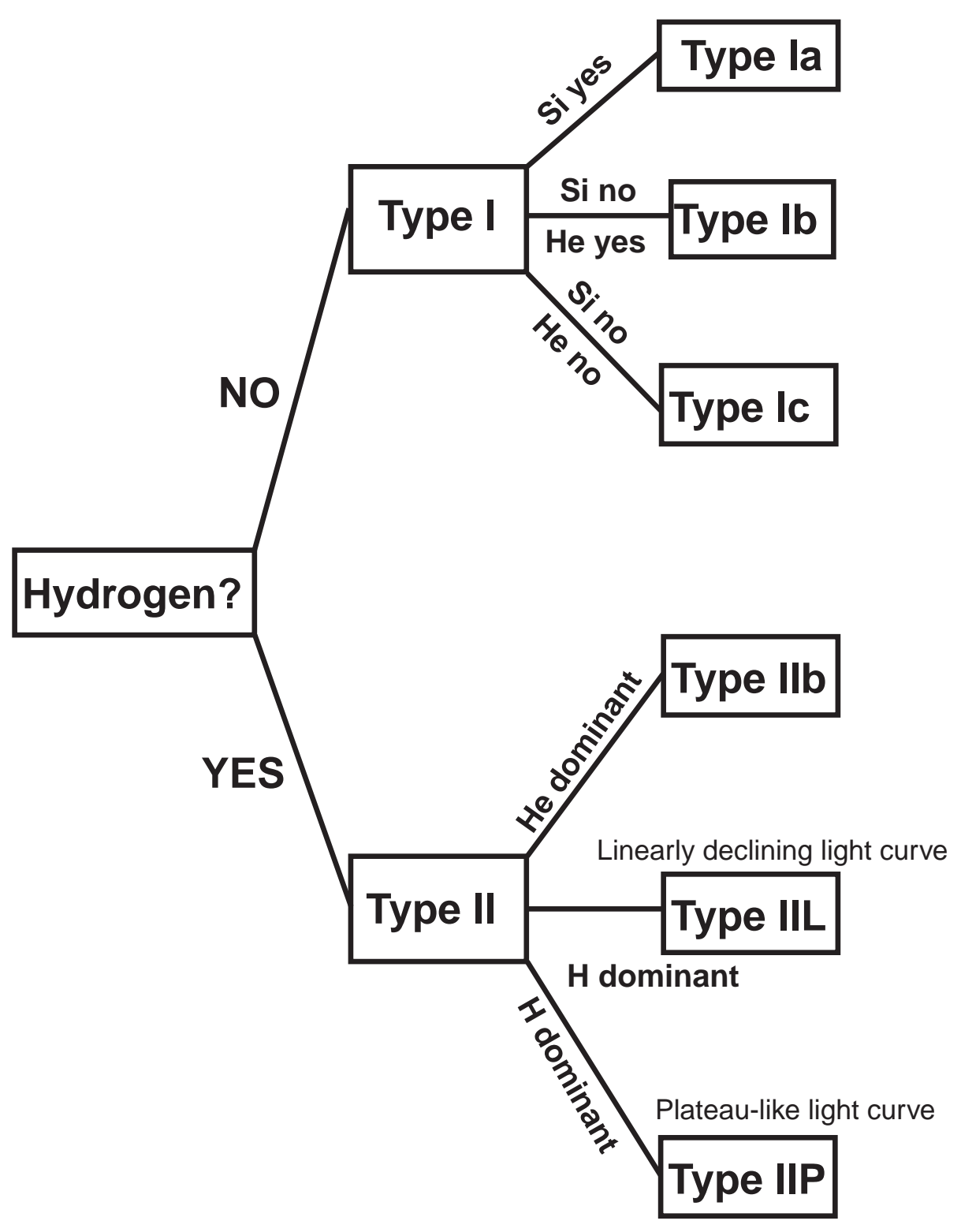

Figure 1: Schematic representation of the major optical classifications of supernovae.

hence have commonly been used as cosmic distance indicators. However, at the detection limit of current radio interferometers, no radio emission has yet been detected from any extragalactic Type-Ia supernovae despite numerous deep searches [17].

Both Type-Ib/c and Type-II supernovae originate from the core collapse of massive stars rather than the thermonuclear explosions of a low mass star that is the origin of Type-Ia SNe. These sources are sub-classified due to the presence or absence of optical lines in the early spectra and their optical light curves. In some cases, the spectra of a supernova have been seen to evolve between these classes, a notable example is the case of SN1993J, which evolved from Type-II to Type-Ib/c within its first few weeks. In general, Type-Ib SNe show strong He I absorption, whereas Type-Ic show only weak He I absorption lines. There are no clear differences between these two 
classes observed at radio wavelengths so I will consequently refer to these sources as Type-Ib/c sources. Other core-collapse SNe, which show hydrogen lines in their early spectra, are Type-II sources. These are commonly divided into Type-IIL and Type-IIP supernovae depending on the shape of their optical light curves. Type-IIL SNe show an approximately linearly declining optical light curve whereas Type-IIP sources display a plateau-like optical light curve. The radio SN1979C and SN1987A provide examples of each of these sub-classes, respectively. At radio wavelengths, several examples of each of these core-collapse supernovae events have been detected (see [18] and references therein).

\subsection{Detections of optical and radio supernovae}

At present, discoveries of approximately $3 \rightarrow 4$ hundred extragalactic supernovae are reported each year. Almost all of these objects are detected at optical wavelengths with the majority of these being reported by professional, large-scale supernovae search programmes, such as KAIT [19], however some are still discovered by amateur astronomers. At radio wavelengths, relatively few of these optically identified objects are ever detected. To date, well in excess of 100 supernovae have lower limits to their radio emission established, whilst only several dozen sources out of the thousands of optical supernovae have been detected and had detailed observations of their radio evolution made. Of these radio detected supernovae, all are core-collapse sources (Type-Ib/c or Type-II). Of the radio detections so far made, the following broad characteristics have been established:

- The radio emission from Type-Ia sources falls below the current sensitivity limit of radio interferometers such as the VLA.

- Type-Ib/c supernovae can be radio luminous. They have rapid turn-ons/turn-offs at radio frequencies, peaking at centimetric wavelengths on comparable timescales to their optical emission peak. Their radio spectra are steep $\left(\alpha \lesssim 1\left[\mathrm{~S} \propto v^{-\alpha}\right]\right)$.

- The radio emission from Type-II supernovae shows very varied properties, including a wide variations in luminosity and timescales. The spectral indices of Type-II SNe have a tendency to be flatter and they tend to have a slower turn-on and can be more long lived than Type-Ib/c sources.

For further details on the radio properties of SNe please see some of the many excellent and comprehensive reviews, for example Weiler et al. [18].

Whilst these characteristics are established for the optically identified and classified supernovae that have been studied in detail at radio wavelengths, there are many compact radio sources, many of which are thought to be supernovae. Of these sources detected in the centres of dustobscured galaxies, a large fraction are never optically classified. It is assumed that many of them are massive core-collapse supernovae; extreme examples of these sources may be the those found in the centre of Arp220 [5, 20, 21, 22, 23, or the luminous radio supernovae detected in NGC7469 [24, 25] (see Fig2). 


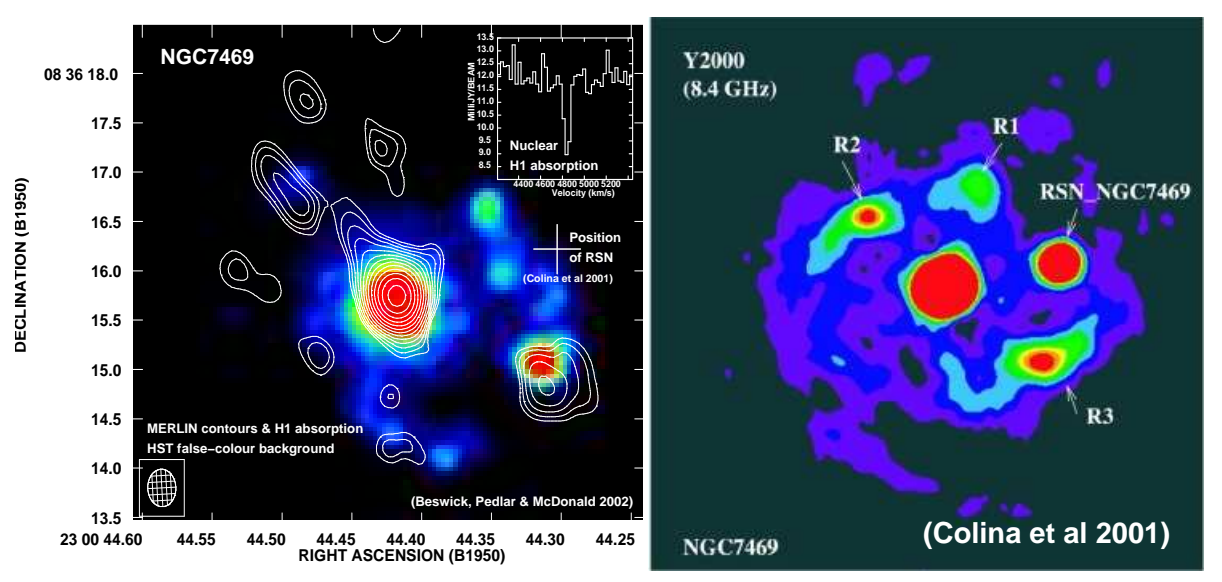

Figure 2: 'Before' and 'after' radio images of the centre of the nearby Seyfert galaxy NGC7469. In the left-hand panel, a MERLIN radio contoured map is shown overlaid upon a HST FTC2 false colour image (from Beswick, Pedlar \& McDonald 2002 [26]). This radio image was taken before SN2000ft was detected. The right-hand panel shows an 8.4-GHz VLA map of the same region, observed following the explosion of SN2000ft. The new bright source to the west of the nucleus, situated in the well-known cirumnuclear starburst ring, is interpreted as a bright radio supernova [24, 25].

\section{Detecting and monitoring supernovae at radio wavelengths}

The multiwavelength detection and monitoring of the radio emission from supernova events provides a wealth of information about the density and structure of the circumstellar medium (CSM). From the modelling of these radio 'light' curves, it is possible to derive evidence about the clumpiness or filamentary nature of the stellar wind prior to the star becoming a supernova, the mass-loss history of the progenitor star, possible binary companions of the progenitor as well as constraining the explosion date for the supernova event (see Weiler et al. [18] for a review of this work and references therein).

The radio 'light' curve of a supernova goes through an initial rise, following the explosion, as the material surrounding the star becomes less dense and opaque as it expands. This material becomes more transparent at higher frequencies first, so the explosion becomes visible at higher frequencies before the light curve rises at lower frequencies. As this expanding envelope grows larger, it cools. At a certain point, the material becomes cool enough that it no longer radiates and, at optical wavelengths, the source fades. For core-collapse radio supernovae, the radio emission is thought to originate from an approximately spherical expanding shell just behind the blast-wave interaction with the CSM. This radio emission is non-thermal synchrotron emission originating from the acceleration of free electrons in the enhanced magnetic field arising from the shock wave interaction of the blast-wave and the CSM. As a consequence, the surrounding ambient conditions of the CSM dictate the evolution of the supernovae radio emission.

\subsection{One nearby example: The Type-IIP Supernova 2004dj in NGC2403}

The supernova 2004dj was detected, visually on 31 Jul. 2004 [27] and at radio wavelengths using the VLA at $8.4 \mathrm{GHz}$ on 2 Aug. 2004 [28], providing an ideal opportunity to study a nearby supernova and its evolution at radio wavelengths. Peaking at $11.2 \mathrm{mag}$. SN 2004dj was the brightest 


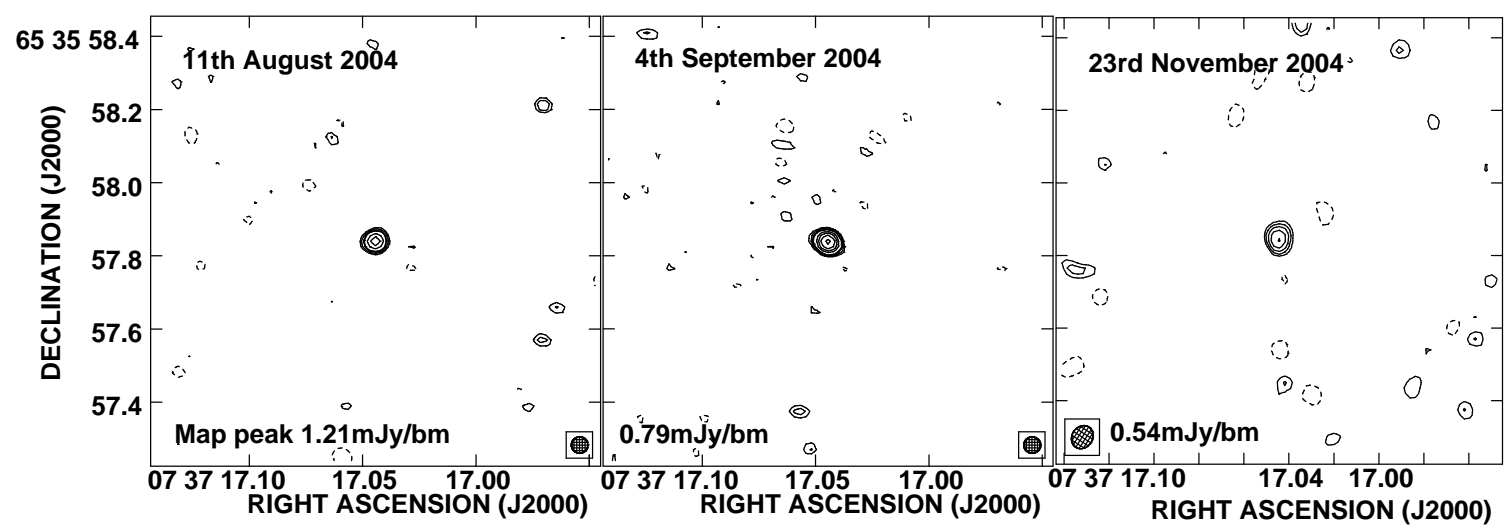

Figure 3: Images of the faint radio emission from the optically bright nearby supernova SN 2004dj in NGC2403 from Beswick et al.[35].

optical supernova for several years. Shortly after the initial detection, it was reported that the spectrum of SN 2004dj showed features typical of Type II-P supernovae [29]. Type II-P supernovae are believed to originate via core collapse in hydrogen-rich, massive stars.

At radio wavelengths, SN2004dj was detected only a few days following the optical discovery. MERLIN observations using a subset of the array began in early August 2004 and continued into early October, followed by imaging runs using the full array in November and December 2004 (Fig. 3). This allowed a detailed 5-GHz 'light' curve to be determined (Fig. Đ).

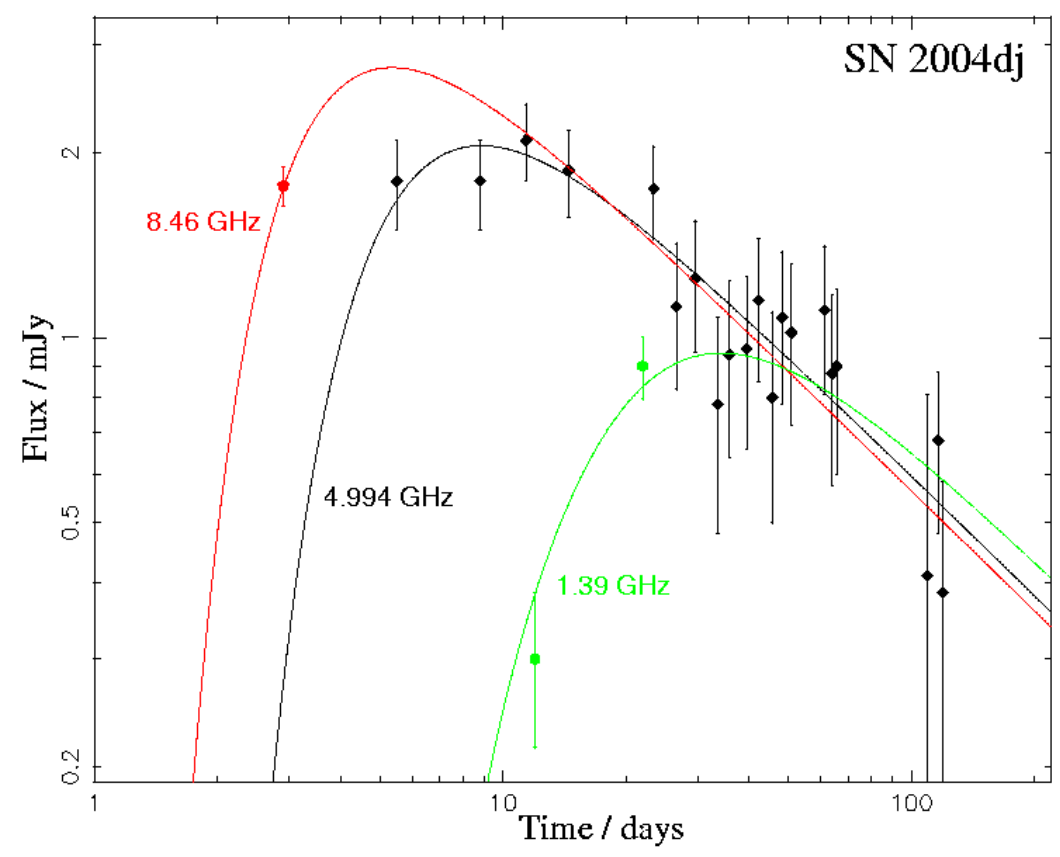

Figure 4: The early radio 'light' curve of the bright nearby supernova SN 2004dj in NGC2403 from 35 , 37, 36].

These MERLIN observations allowed the position of the source to be determined to an accuracy of better than 50 mas [30], coincident with the optical [27] and Chandra X-ray [31] positions, 


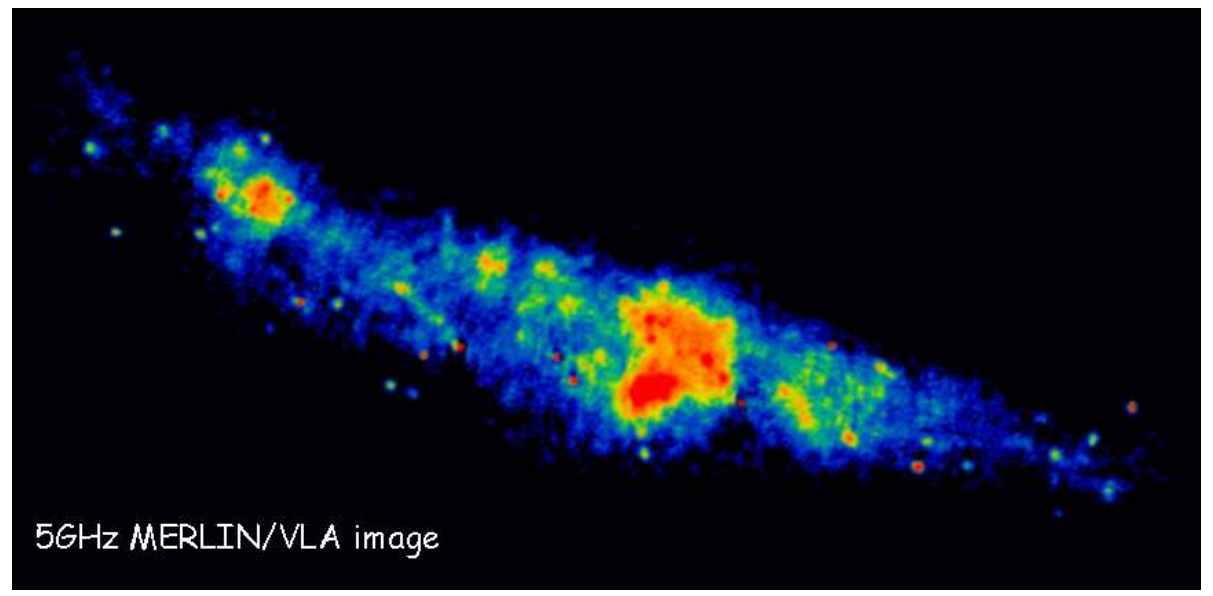

Figure 5: False colour combined MERLIN+VLA 5-GHz image of M82 showing numerous compact radio sources embedded within the diffuse radio continuum. MERLIN and VLBI observations resolve all of these compact sources (Courtesy of Tom Muxlow).

and that of the star cluster n2403-2866 [32]. This illustrates the usefulness of high-resolution observations, for example using MERLIN, for this kind of programme. SN2004dj was also observed with the VLA [28], which at the time was in its most compact configuration, the resulting measured position was affected by extended emission from the galaxy and lies $\sim 1$ "' 2 from other measurements.

Supernova 2004dj was optically classified as Type II-P [29], a relatively common type of supernova optically, but rarely detected at radio wavelengths. In fact, prior to $2004 \mathrm{dj}$, the only two Type II-P SNe detected by radio telescopes were SN 1999em [33], for which no light curve was established, and the well-observed SN 1987A [34], both of which were also relatively weak radio emitters. With the exception of the LMC supernova 1987A, observations of SN2004dj provide the only detailed information regarding the radio 'light' curve of this class of core-collapse supernova.

\section{Radio imaging of nearby supernovae and supernova remnants}

\subsection{Supernova remnants in M82}

As has previously been mentioned, the central starburst region within the nearby irregular galaxy M82 contains a wealth of compact sources that are the consequence of the massive and ongoing star-formation within the centre of this source. Early high-resolution radio observations by Unger et al. [1] and Kronberg et al. [2, 仙 identified these sources as population of supernovae and supernova remnants. Later, more detailed work ([3, 5]) have shown that these sources are a mixture of supernova remnants and compact HII regions.

Over the $\sim 20$ years, high-resolution VLBI and MERLIN observations have been used to resolve and monitor the structural evolution of these sources in great detail [38, 39, 3, 40, 41, 42, 43, [44, 45] (see Fig. 5).

The two most compact sources in M82 $(41.95+475$ and 43.31+592) have been resolved in detail using a series of global VLBI observations over the last 20 years [41, 42, 44, 45]. Three 18-cm global VLBI images of the most compact source in M82 are shown in Fig. 6. This series 


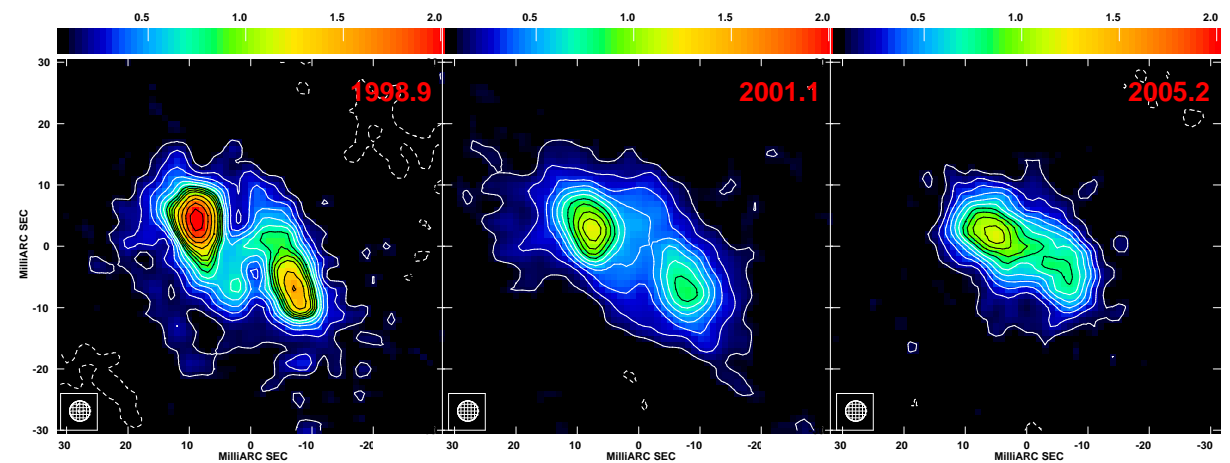

Figure 6: Contour images of the compact radio source $41.95+575$ from the global VLBI epochs observed on 28 Nov 1998, 23 Feb 2001 and 3 Mar 2005. Each of these images has been convolved with a 3.3-mas circular beam. The three epochs are contoured with linear multiples $(1,1,2, \ldots 10) \times 0.21,0.11$ and $0.11 \mathrm{mJy}$ beam $^{-1}$, respectively. Images are taken from [42, 44, 45].



Figure 7: Contour images from all five VLBI epochs of the compact RSN 43.31+592. From left to right these observations were made on 11 Dec. 1986, 2 Jun. 1997, 28 Nov. 1998, 23 Feb. 2001 and 3 Mar. 2005, respectively. Maps of all five epochs have been convolved with a circular beam of 15 mas, to match the resolution of earlier EVN-only images in 1986 and 1997. Maps for all five images are contoured with 1, 1, $2,3,4,5,6,7,8,9$ and $10 \times 0.35 \mathrm{mJy}_{\text {beam }}{ }^{-1}$. On the fourth, three crosses are plotted to show the relative Gaussian-fitted positions of the three components in epoch 1. Images are taken from [42, 44, 45].

of 4-mas angular resolution images observed in 1998, 2001 and 2005 show evolving radio structure of $41.94+475$. Although initially considered a compact radio supernova remnant, similar to many of the other sources in M82, 41.9+475 displays a distinctly different radio structure. The compact VLBI structure of this source appears to be bipolar in nature with two 'hot-spots'. These two components appear to be moving apart quite slowly with expansion velocities in the range of only $\sim 1000$ to $2000 \mathrm{~km} \mathrm{~s}^{-1}$ between 1998 and 2001, although the most recent epoch shows considerable further structural evolution making a definitive velocity calculation hard to achieve (see [45]). Whilst it is clear that $41.95+475$ is evolving both in its radio flux density and milliarcsecond radio structure, it is still unclear as to the true nature of this source. It has recently been speculated that this source is not a true radio supernova event but something more exotic, such as the remnant of an off-axis GRB (see [43, 44, 45]).

Unlike 41.95+475, the second most compact radio source in M82, 43.31+592, resembles the classical shape for a radio supernova remnant. As can be seen in Fig. ㄱ. 43.31+47 shows a regular ring-like structure with breakout region toward the north-eastern corner. Over the 20 years of monitoring, the radio structure and size of this remnant have been studied in detail. These observations have shown that this source is in near free expansion with a velocity of between 9000 and 


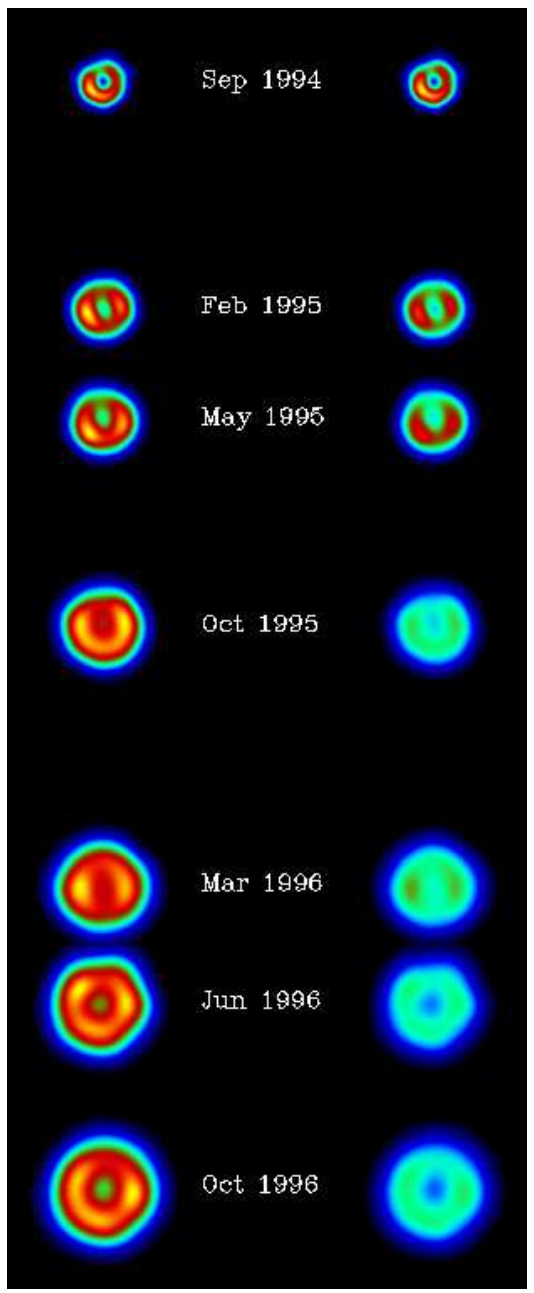

Figure 8: Global VLBI images showing the first few years of the expansion of SN 1993J in M81 [53, 54].

$11000 \mathrm{~km} \mathrm{~s}^{-1}$ [41, 42, 44]. This value is close agreement with other radio expansion measurements of this source made using MERLIN by Muxlow et al. [46]. Both of these sources along with several of the other compact radio supernova remnants in M82 are discussed in more detail elsewhere in this proceedings [45].

\subsection{SN1993J in M81}

Of all supernovae observed with high resolution VLBI, SN1993J in M81 has been subject to the most detailed scrutiny. SN1993J exploded on 28 Mar 1993 and was first detected at radio wavelengths just a few days later at the beginning of April 1993 [47, 48]. By virtue of its closeness (M81 is just 3.6 Mpc away), its brightness (at the time the nearest northern hemisphere supernova since 1937), and its high northern declination, this source an ideal target for VLBI observations. The source was first detected using VLBI on 25 Apr. 1993 and these observations established the position of the supernova to an accuracy of a few milliarcsec [49, 50].

Early size estimates of SN1993J were established approximately 30 days following the supernova explosion implying an angular size of $0.28 \pm 0.06$ mas on 1 May 1993 [51]. Following this, a 


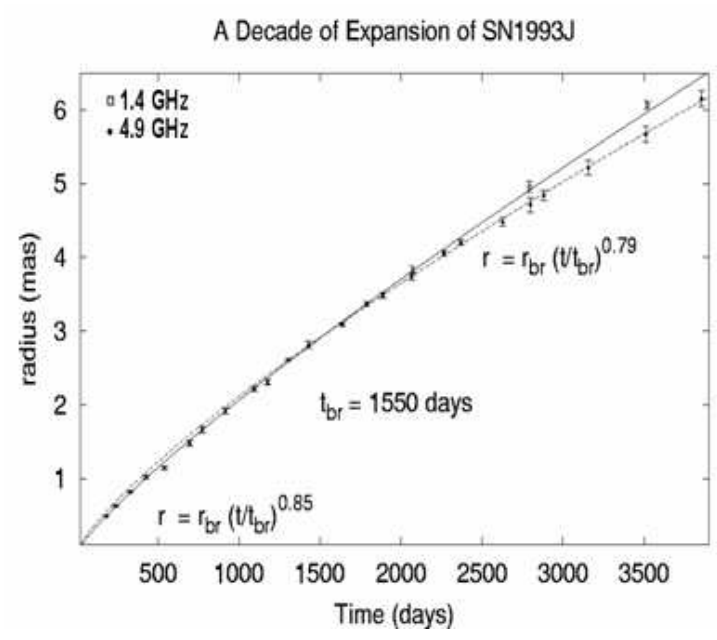

Figure 9: A decade of expansion of SN1993J. Plot of size measurements derived from VLBI observations for the expanding shell of SN1993J showing the expansion to be decelerating [55].

series of VLBI measurements of the sources structure and expansion have been made [52, 53, 54]. The initial expansion rate of the radio emission was $\sim 2.4 \mu \mathrm{arcsec} \mathrm{day}^{-1}$. Subsequent VLBI imaging over more than a decade has resolved SN1993J into a self-similarly expanding radio shell (see Fig. (8) with a recorded deceleration parameter of between 0.79 and $0.9\left(\theta \propto \mathrm{t}^{m}\right)$. After approximately day 1550, an increased deceleration was observed at $6 \mathrm{~cm}$.

Very recent results from Marcaide et al. [55] conclude that the expansion of SN1993J is actually self-similar, even if at $6 \mathrm{~cm}$ there appears to be increased deceleration after day $\sim 1500$ (see Fig. 9). This kink in the expansion curve can be explained by a combination of the partial lifting of the absorption of the emission from the back side of the shell behind the ionized ejecta and a pronounced radial drop of the magnetic field biasing the measurement of the ring size.

Due to the immense detail, in which it has been possible to study SN1993J at radio wavelengths, numerous articles have been written (many of which are not mentioned here). For a more detailed precis of the impact of the observations of SN1993J, please refer to the numerous articles published in Cosmic Explosions: On the tenth anniversary of SN1993J IAU colloquium192, Eds. Marcaide \& Weiler.

\subsection{SN2004et in NGC6946}

In September 2004, a bright supernova, 2004et, was discovered optically [56]. Its host galaxy, NGC 6946, is a nearby active starburst galaxy, which has hosted many historical supernovae. Initially, it was hoped that this nearby, optically luminous, Type-II supernova would also become luminous at radio wavelengths, providing an opportunity, similar to that of SN1993J, to observe its radio structural evolution in great detail. However, following early radio monitoring using both MERLIN and the VLA [57, 36], it became apparent that the radio emission of SN2004et would not reach a high luminosity. The early light-curve monitoring of this source recorded a peak flux density of $\sim 2.5 \mathrm{mJy}^{\text {beam }}{ }^{-1}$ at $5 \mathrm{GHz}$ at an age of approximately 40 days. However, a first epoch of sensitive 8.4-GHz global VLBI observations were made by Marti-Vidal et al. [58] on $20 \mathrm{Feb}$ 2005 (day 153). These observations detected and marginally resolved SN2004et (Fig. 10). 


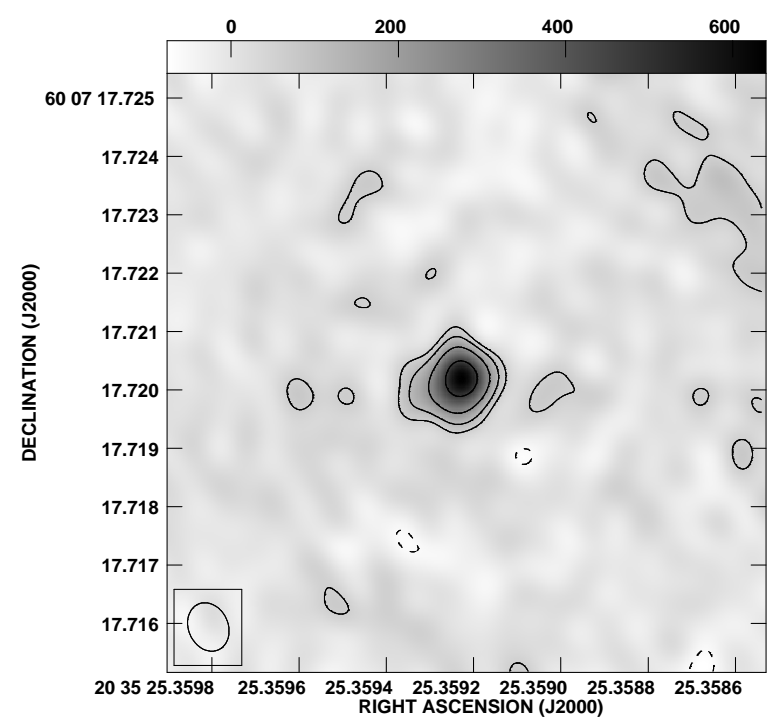

Figure 10: Global VLBI image of SN2004et on day 153 (from Marti-Vidal et al. [58]). Map peak flux is $0.639 \mathrm{mJy} / \mathrm{bm}$ and convolved is with a $0.86 \times 0.68$ mas beam.

The radio structure of SN2004et shows a bipolar geometry, which is interpreted as either coming from a shell with two hot spots or, an ejecta that is expanding anisotropically. Favouring the former of these interpretations an expansion velocity of $16000 \pm 2000 \mathrm{~km} \mathrm{~s}^{-1}$ is derived. This velocity is comparable to the early expansion velocities recorded for other similar radio supernovae.

\subsection{Optically undetected radio supernovae}

In general, this review has concentrated on a few examples of extragalactic radio supernovae and supernova remnants that have been well studied at radio wavelengths. However, as mentioned to earlier, there are several star-forming galaxies where radio observations, and in particular VLBI observations, are now beginning to reveal large numbers of compact radio components, which are thought to be powerful radio supernovae or supernova remnants. One example of this is the nearby ultra-luminous infrared galaxy Arp220. Within the centre of Arp220, 50 compact milliarcsecondscale radio components have been detected by Smith et al [5], Rovilos et al. [20] and Lonsdale et al. [21]. As can be seen in Fig. 11, these numerous compact components are buried within the central starburst of Arp220 and have required the extremely high resolution and high sensitivity of current VLBI arrays in order to detect them. More recently, and reported in this meeting by Parra et al. [22, 23], multi-frequency VLBI observations along with long-term monitoring [59] of these sources has begun to confirm them to be a combination of powerful radio supernovae and supernova remnants.

High-resolution radio observations of objects, such as Arp220 and M82, remain one of the few ways in which the supernovae and remnants with these dust, highly-obscured starburst galaxies can be explored, further underlining the necessity of radio observations of supernovae.

\section{Summary and future work}

This, somewhat incomplete, review of radio supernova and their remnants has made some 


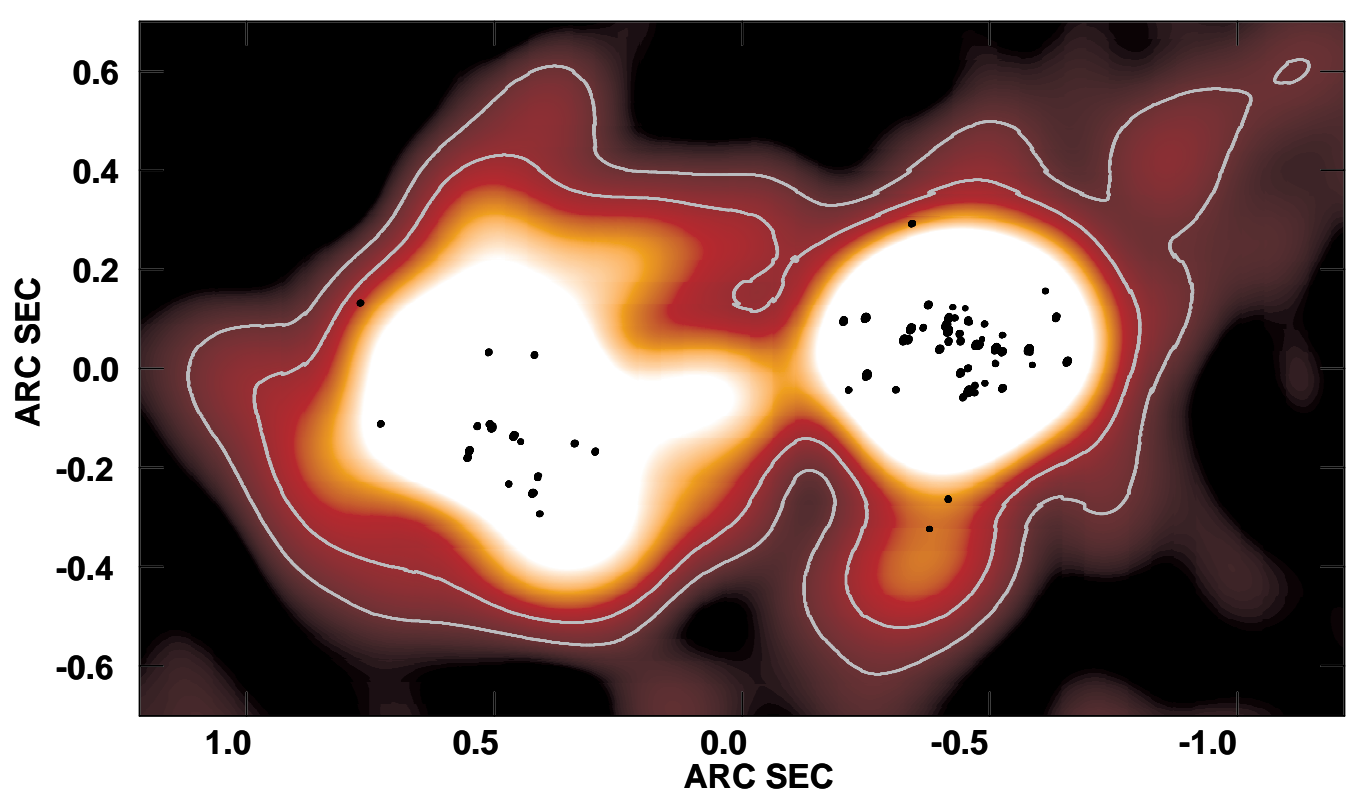

Figure 11: Extremely deep 1.6-GHz global VLBI image of the centre of Arp220. The small black dots show the high resolution VLBI sources (Lonsdale et al. [21]). This VLBI image is overlaid upon a 'lower' resolution MERLIN 1.4-GHz image of the central region of Arp220 from Mundell, Ferruit \& Pedlar [64].

efforts to report a few of the highlights of radio, and in particular VLBI observations, of supernovae. It should be noted, however, that several other supernovae have also been extensively observed. In particular, these are: SN1973C [60, 61], SN1986J [62, 63] and 1987A. Each of these sources has been subject massively important individual studies, for which there is not space here to fully do them justice.

In summary, whilst there has been a vast amount learnt about radio supernovae, radio supernova remnants, the CSM surrounding radio supernovae and their progenitors via both radio flux density monitoring and detailed VLBI imaging, to date only a relatively small fraction of supernovae have been studied at these wavelengths. It is clear that only a few supernovae detected optically are radio emitters at levels that can be studied with current instruments, which naturally limits the number of objects that can presently be studied in detail. However, considering vast amounts of information that can be obtained even from relatively few objects, I can only describe the future of this somewhat explosive subject as luminous! This is specially true considering the advent of new more sensitive radio arrays, such as the EVLA and e-MERLIN, along with the more rapid response of high sensitive VLBI arrays through real-time observations (eVLBI).

\section{Acknowledgments}

I would like to thank Phil Diamond, Tom Muxlow, Alan Pedlar, Megan Argo, Danielle Fenech, Jon Marcaide, Ivan Marti-Vidal and Rodrigeo Parra for their many invaluable contributions to this review. 


\section{References}

[1] S. W. Unger, A. Pedlar, D. J. Axon, P. N. Wilkinson, P. N. Appleton, Young supernovae in the starburst galaxy M82, MNRAS, 1984, (211), 783

[2] P. P. Kronberg, P. N. Wilkinson, High-resolution, multifrequency radio observations of M82,ApJ 1975 (300) 430

[3] T.W.B. Muxlow, A. Pedlar, P.N. Wilkinson, D.J. Axon, E.M. Sanders, A.G. de Bruyn, The Structure of Young Supernova Remnants in M82, MNRAS 1994 (266) 455

[4] P. P. Kronberg, P. Biermann, F. R. Schwab, The nucleus of M82 at radio and X-ray bands - Discovery of a new radio population of supernova candidates, ApJ 1985 (291) 693

[5] Harding E. Smith, Carol J. Lonsdale, Colin J. Lonsdale, Philip J. Diamond, A Starburst Revealed-Luminous Radio Supernovae in the Nuclei of ARP 220, ApJ, 1998, (493), L17

[6] A. R. McDonald, T. W. B. Muxlow, K. A. Wills, A. Pedlar, R. J. Beswick, A parsec-scale study of the 5/15-GHz spectral indices of the compact radio sources in M82, MNRAS, 2002, (334), 912

[7] Y. M. Pihlström, J. E. Conway, R. S. Booth, P. J. Diamond, A. G. Polatidis, EVN and MERLIN observations of III Zw 35 . A starburst continuum and an $\mathrm{OH}$ maser ring, A\&A, 2001, (377), 413

[8] Susan G. Neff, James S. Ulvestad, Stacy H. Teng, A Supernova Factory in the Merger System Arp 299, ApJ, 611, 186

[9] C. L. Carilli, G. B. Taylor, The Extreme Compact Starburst in Markarian 273, ApJ, 2000, (532L), 95

[10] M. Bondi, M-A. Pèrez-Torres, D. Dallacasa, T. W. B. Muxlow, A supernova factory in Mrk 273?, MNRAS, 2005, (361), 748

[11] Jack F. Gallimore, Robert J. Beswick, Parsec-Scale Radio Structure of the Double Active Nucleus of NGC 6240, AJ, 2004, (127), 239

[12] Bruno Leibundgut, Type Ia Supernovae, Astronomy and Astrophysics Review, 2000, (10), 179

[13] David Branch, Type IA Supernovae and the Hubble Constant, Annual Review of Astronomy and Astrophysics, 1998, (36), 17

[14] T. J. O’Brien, M. F. Bode, R. W. Porcas, T. W. B. Muxlow, S. P. S. Eyres, R. J. Beswick, S. T. Garrington, R. J. Davis, A. Evans An asymmetric shock wave in the 2006 outburst of the recurrent nova RS Ophiuchi, Nature, 2006, 442, 279

[15] T. J. O’Brien, M.F. Bode, R.W. Porcas, T.W.B. Muxlow, R.J. Beswick, S.T. Garrington, S.P.S. Eyres, J.P. Osborne, K.L. Page, A.P. Beardmore, M.R. Goad, S. Starrfield, J-U. Ness, A. Evans, G.K. Skinner, R.J. Davis The 2006 outburst of RS Ophiuchi, PoS, 2006 This proceedings (PoS(8thEVN)052)

[16] J. L. Sokoloski, G. J. M. Luna, K. Mukai, Scott J. Kenyon, An X-ray-emitting blast wave from the recurrent nova RS Ophiuchi, Nature, 2006, 442, 276

[17] Nino Panagia, Schuyler D. Van Dyk, Kurt W. Weiler, Richard A. Sramek, Christopher J. Stockdale, Kimberly P. Murata, A Search for Radio Emission from Type Ia Supernovae, ApJ, 2006, (646), 369

[18] Kurt W. Weiler, Nino Panagia, Marcos J. Montes, Richard A. Sramek, Radio Emission from Supernovae and Gamma-Ray Bursters, Annual Review of Astronomy and Astrophysics, 2002, (40), 387 
[19] Alexei V. Filippenko, W. D. Li, R. R. Treffers, Maryam Modjaz, The Lick Observatory Supernova Search with the Katzman Automatic Imaging Telescope, Small Telescope Astronomy on Global Scales, ASP Conference Series Vol. 246, IAU Colloquium 183. Edited by Bohdan Paczynski, Wen-Ping Chen, and Claudia Lemme. San Francisco: Astronomical Society of the Pacific, 2001, (246), 121

[20] E. Rovilos, P. J. Diamond, C. J. Lonsdale, H. E. Smith, C. J. Lonsdale, The 18-cm light curves of the luminous radio supernova candidates in Arp 220, MNRAS, 2005, (359) 827

[21] Colin J. Lonsdale, Philip J. Diamond, Hannah Thrall, Harding E. Smith, Carol J. Lonsdale, VLBI Images of 49 Radio Supernovae in Arp 220, ApJ, 2006, (647), 185

[22] Rodrigo Parra, John E. Conway, Phillip J. Diamond, Hannah Thrall, Colin J. Lonsdale, Carol J. Lonsdale, Harding E. Smith, The radio spectra of the compact sources in Arp220: A mixed population of supernovae and supernova remnants, 2006 Submitted to ApJ.

[23] Rodrigo Parra, John E. Conway, Phillip J. Diamond, Hannah Thrall, Colin J. Lonsdale, Carol J. Lonsdale, Harding E. Smith Multiwavelength radio observations of the compact starburst in Arp220, PoS, 2006 This proceedings (PoS(8thEVN)033)

[24] L. Colina, A. Alberdi, J. M. Torrelles, N. Panagia, A. S. Wilson, Discovery of a Bright Radio Supernova in the Circumnuclear Starburst of the Luminous Infrared Seyfert 1 Galaxy NGC 7469, ApJ, 2001, (553L), 19

[25] A. Alberdi, L. Colina, J. M. Torrelles, N. Panagia, A. S. Wilson, S. T. Garrington, S. T., Evolution of the Circumnuclear Radio Supernova SN 2000ft in NGC 7469, ApJ, 2006, (638), 938

[26] R. J. Beswick, A. Pedlar, A. R. McDonald, Sub-arcsecond atomic hydrogen absorption in the Seyfert galaxies NGC 7674 and NGC 7469 MNRAS, 2002, (335), 1091

[27] Nakano, S., Itagaki, K., Bouma, R. J., Lehky, M. \& Hornoch, K, Supernova 2004dj in NGC 2403, IAUC Circular, 2004, (8377), 1

[28] Stockdale, C. J., Sramek, R. A., Weiler, K. W., Van Dyk, S. D., Panagia, N., Pooley, D., Lewin, W. \& Marcaide, J. M., Supernova 2004dj in NGC 2403, IAUC Circular, 2004, (8379), 1

[29] Patat, F., Benetti, S., Pastorello, A. \& Filippenko, A. V., Supernova 2004dj in NGC 2403, IAUC Circular, 2004, (8378), 1

[30] M. K. Argo, T. W. B. Muxlow, R. J. Beswick, A. Pedlar, J. M. Marcaide, Supernova 2004dj in NGC 2403, IAUC Circular, 2004, (8399), 1

[31] D. Pooley, W. H. G. Lewin, Supernova 2004dj in NGC 2403, IAUC Circular, 2004 (8390), 1

[32] S. S. Larsen, T. Richtler, Young massive star clusters in nearby galaxies. I. Identification and general properties of the cluster systems, A\&A, 1999, (345), 59

[33] Pooley, D. et al. X-Ray, Optical, and Radio Observations of the Type II Supernovae 1999em and 1998S, ApJ, 2002, (572), 932

[34] A. J. Turtle, D. Campbell-Wilson, J. D. Bunton, D. L. Jauncey, M. J. Kesteven, A prompt radio burst from supernova 1987A in the Large Magellanic Cloud 1987, Nature, 1987, (327), 38

[35] R. J. Beswick, T. W. B. Muxlow, M. K. Argo, A. Pedlar, J. M. Marcaide, K. A. Wills, Monitoring of the Prompt Radio Emission from the Unusual Supernova SN 2004dj in NGC 2403, ApJ, 2005, (623L), 21

[36] M. K. Argo, Radio studies of starburst galaxies, PhD Thesis, University of Manchester, 2006 
[37] M. K. Argo, R. J. Beswick, T. W. B. Muxlow, A. Pedlar,D. Fenech, H. Thrall, MERLIN monitoring of recent core-collapse supernovae in proceedings of Stellar End Products, Granada, April 13-15, 2005, Memorie S. A. It, 2005, (76), 565

[38] P. N. Wilkinson, A. G. de Bruyn, A VLBI map of 1661 MHz of the brightest radio source in M82, MNRAS, 1984, (211), 593

[39] N. Bartel, M. I. Ratner, A. E. E. Rogers, I. I. Shapiro, R. J. Bonometti, N. L. Cohen, M. V. Gorenstein, J. M. Marcaide, R. A. Preston, VLBI observations of 23 hot spots in the starburst galaxy M82, ApJ, 1987, (323), 505

[40] W.M. Trotman VLBI Observations of 41.9+58: An intermediate-age radio supernova in the starburst nucleus of M82, MSc Thesis, University of Manchester, 1996

[41] A. Pedlar, T.W.B. Muxlow, M.A. Garret, P.J. Diamond, K.A. Wills, P.N. Wilkinson, W. Alef, VLBI observations of supernova remnants in Messier 82, MNRAS, 1999, (307), 761

[42] A.R. McDonald, T.W.B. Muxlow, A. Pedlar, M.A. Garret, K.A. Wills, S.T. Garrington, P. J. Diamond, P. N. Wilkinson, Global Very Long Baseline Interferometry observations of compact radio sources in M82, MNRAS, 2001, (322), 100

[43] T.W.B. Muxlow, A. Pedlar, R.J. Beswick, M.K. Argo, T.J. O'Brien, D. Fenech, W. Trotman, Is 41.95+57.5 in M82 actually an SNR?, in proceedings of Stellar End Products, Granada, April 13-15, 2005, Memorie S. A. It, 2005, (76), 586

[44] R. J. Beswick, J. D. Riley, I. Marti-Vidal, A. Pedlar, T. W. B. Muxlow, A. R. McDonald, K. A. Wills, D. Fenech, M. K. Argo, 15 years of very long baseline interferometry observations of two compact radio sources in Messier 82, MNRAS, 2006, (369), 1221

[45] D. Fenech, T. W. B. Muxlow, R. J. Beswick, A. Pedlar, M. K. Argo, W. M. Trotman, Supernova remnants in the central starburst region of M82, PoS, 2006, This proceedings

[46] T.W.B. Muxlow, A. Pedlar, A. Riley, J. McDonald, R.J. Beswick, K.A. Wills, Deep radio imaging with MERLIN of the supernova remnants in M82, in proceedings of IAU Colloqu im 192, Springer Proceedings in Physics, 2005, (192), 227

[47] K. W. Weiler, R. A. Sramek, S. D. van Dyke, N. Panagia, Supernova 1993J in NGC 3031, IAUC Circular, 1993, (5752), 1

[48] G. G. Pooley, D. A. Green, Ryle Telescope Observations of Supernova 1993J at 15-GHz - the First 115 Days, MNRAS, 1993, (264L), 17

[49] J. M. Marcaide, A. E. E. Rogers, A. Alberdi, J. C. Guirado, A. Rius, P. Elosegui, I. I. Shapiro, E. Perez, A. R. Whitney, Supernova 1993J in NGC 3031, IAUC Circular, 1993, (5785), 1

[50] J. M. Marcaide, A. E. E. Rogers, A. Alberdi, J. C. Guirado, J. C. Guirado, A. Rius, P. Elosegui, I. I. Shapiro, E. Perez, A. R. Whitney, Supernova 1993J in NGC 3031, IAUC Circular, 1993, (5820), 2

[51] J. M. Marcaide, A. Alberdi, P. Elosegui, J. C. Guirado, F. Mantovani, E. Perez, M. I. Ratner, A. Rius, A. E. E. Rogers, B. P. Schmidt, I. I. Shapiro, A. R. Whitney, Radio-size estimates of SN 1993J, ApJ, 1994, (424L), 25

[52] N. Bartel, M. F. Bietenholz, M. P. Rupen, J. E. Conway, A. J. Beasley, R. A. Sramek, J. D. Romney, M. A. Titus, D. A. Graham, V. I. Altunin, D. L. Jones, A. Rius, T. Venturi, G. Umana, R. L. Francis, M. L. McCall, M. G. Richer, C. C. Stevenson, K. W. Weiler, S. D. van Dyk, N. Panagia, W. H. Cannon, J. Popelar, R. J. Davis, The Shape Expansion Rate and Distance of Supernova $1993 \mathrm{~J}$ from VLBI Measurements, Nature, 1994, (368), 610 
[53] J. M. Marcaide, A. Alberdi, E. Ros, P. Diamond, B. Schmidt, I. I. Shapiro, L. Baath, R. J. Davis, A. G. de Bruyn, P. Elosegui, J. G. Guirado, D. L. Jones, T. P. Krichbaum, F. Mantovani, R. A. Preston, M. I. Ratner, A. Ruis, A. E. E. Rogers, R. T. Schilizzi, C. Trigilio, A. R. Whitney, A. Witzel, A. Zensus, Discovery of Shell-Like Radio-Structure in Supernova 1993J, Nature, 1994, (373), 44

[54] J. M. Marcaide, A. Alberdi, E. Ros, P. Diamond, I. I. Shapiro, J. C. Guirado, D. L. Jones, T. P. Krichbaum, F. Mantovani, R. A. Preston, A. Rius, R. T. Schilizzi, C. Trigilio, A. R. Whitney, A. Witzel, A., Expansion of Supernova 1993J, Science, 1995, (270), 1475

[55] J. M. Marcaide et al. Submitted to ApJ, 2006

[56] T. Zwitter, U. Munari, S. Moretti, Supernova 2004et in NGC 6946, IAUC Circular, 2004, (8413), 1

[57] R. J. Beswick, T. W. B. Muxlow, M. K. Argo, A. Pedlar, J. M. Marcaide, Supernova 2004et in NGC 6946, IAUC Circular, 2004, (8435), 3

[58] I. Marti-Vidal, J. M. Marcaide, A. Alberdi, J. C. Guirado, L. Lara, M. A. Pèrez-Torres, E. Ros, M. K. Argo, R. J. Beswick, T. W. B. Muxlow, A. pedlar, I. I. Shapiro, C. J. Stockdale, R. A. Sramek, K. W. Weiler, 8.4GHz VLBI Observations of SN2004et, A\&A 2007, (470), 1071

[59] Hannah Thrall et al., In preparation

[60] N. Bartel, A. E. E. Rogers, I. I. Shapiro, M. V. Gorenstein, C. R. Gwinn, J. M. Marcaide, K. W. Weiler, Hubble's constant determined using very-long baseline interferometry of a supernova, Nature, 1985, (318), 25

[61] Norbert Bartel, Michael F. Bietenholz, SN 1979C VLBI: 22 Years of Almost Free Expansion, ApJ, 2003, (591), 301

[62] M. F. Bietenholz, N. Bartel, M. P. Rupen, SN 1986J VLBI: The Evolution and Deceleration of the Complex Source and a Search for a Pulsar Nebula, ApJ, 2002, (581), 1132

[63] N. Bartel, M. P. Rupen, I. I. Shapiro, R. A. Preston, A. Rius, A high-resolution radio image of a young supernova, Nature, 1991, (350), 212

[64] C. G. Mundell, P. Ferruit, A. Pedlar, Nuclear Gasdynamics in Arp 220: Subkiloparsec-Scale Atomic Hydrogen Disks, ApJ, 2001, (560), 168 